\title{
Editorial
}

\author{
Alexander Zauner
}

Online veröffentlicht: 11. November 2009

(C) Springer-Verlag 2009

\section{Sehr geehrte Leserinnen und Leser,}

für die Zeitschrift der markt - Journal für Marketing hat 2009 eine neue Ära begonnen. Die strategische Partnerschaft der Österreichischen Gesellschaft für Absatzwirtschaft (ÖGA) mit dem renommierten SpringerVerlag hat Qualität, Reichweite und Reputation von der markt grundlegend erhöht. Als erster eindrucksvoller Beweis dafür ist das von Prof. Rudolf R. Sinkovics edierte Special Issue zu „Qualitativer Marketing Forschung“ anzusehen. Die Erweiterung des Editorial Boards durch anerkannte Wissenschaftler, zusätzliche Schwerpunktausgaben zu aktuellen Themen, die Stärkung des Mittel-Ost-Europa-Bezuges, aber auch die Möglichkeit, die neuesten Beiträge vor Erscheinen des Heftes online zu konsultieren, sowie umfassende Recherchemöglichkeiten in SpringerLink (http:// www.springerlink.com) sind weitere positive Auswirkungen dieser Kooperation.

In diesem Sinne widmet sich die Ausgabe 2009/3 von der markt einer Reihe hoch interessanter und wissenschaftlich anspruchsvoller Themen:

Im ersten Aufsatz von Heribert Gierl und Yajing Zhu wird die in der Marketingliteratur wenig erforschte Möglichkeit untersucht, Einfluss darauf zu nehmen, auf welche Ursache der Kunde einen Fehler zurückführt. Die Autoren übertragen dazu Aussagen von Attributions- und von Gerechtigkeitstheorien auf Fehler in Anbieter-Kunden-Beziehungen und entwickeln ein attributionstheoretisches Dreieck.
Oliver Schwarz behandelt im zweiten Aufsatz den Net Promoter Score (NPS) als Instrument zur Messung der Kundenloyalität und der Kundenzufriedenheit, sowie als Indikator für nachhaltiges Wachstum. Basierend auf Daten aus Kundenbefragungen der deutschen Direktbank IngDiBa werden im Rahmen der Arbeit Konfidenzintervalle für den NPS mit Hilfe des Bootstrap Verfahrens ermittelt.

Im dritten Artikel untersuchen Gregor Jawecki, Johann Füller und Kurt Matzler wie Personen in virtuellen Gemeinschaften Modifikationen für bestehende Produkte und Neuprodukte entwickeln. Dies wird anhand von Chinesisch- und Englischsprachigen Online Communities zum Thema Basketballschuhe beleuchtet.

In dem Book Review von Irene Knava wird das erst kürzlich erschienene Buch zu „Audiencing“ ausführlich besprochen.

Zum Abschluss enthält diese Ausgabe von der markt - Journal für Marketing einen Call for Papers zu einem Special Issue mit dem Thema „Loyalty Programs von Banken und Versicherungen“. Nach „Measurement and Methodological Problems in Marketing Research“, „Qualitativer Marketing Forschung“ und „Neuromarketing“ folgt auch dieses für Frühling 2011 geplante Special Issue der Tradition, innovativen Themen eine Plattform in der markt zu geben.

Alexander Zauner

\section{A. Zauner (}

WU Wien,

Department für Marketing, Institut für Marketing-Management, Augasse 2-6, 1090 Wien, Österreich E-Mail: alexander.zauner@wu.ac.at 